\title{
Thermodynamic and Thermophysical Properties of Dry Air by Using Cubic Peng- Robinson EoS for Gas Mixtures
}

\author{
Mustafa Turhan Çoban ${ }^{1}$, Süleyman Kavas ${ }^{2 *}$ \\ School of Engineering, Department of Mechanical Engineering; Ege University, Izmir, Turkey \\ Doğu HVAC Systems R\&D Center, ITOB Organized Industry Zone, 10001 Sk. No:435473, Izmir, Turkey \\ E-mail: 1 turhan_coban@yahoo.com
}

Received 14 June 2020, Revised 12 August 2020, Accepted 14 August 2020

\begin{abstract}
Dry air is widely used in industrial and technological applications. Ideal gas EoS is used in dry air thermodynamic property calculations. For most applications, it might be sufficient, but when applications with higher pressure zones are considered, error level will increase. Peng-Robinson cubic Eq. of states is considered for better accuracy of the thermodynamic properties for dry air in this paper. So, the objective of this study is to suggest a more accurate EoS for thermodynamic and heat transfer analyses. Set of computer programs were developed in java language to calculate thermodynamic properties like specific volume, internal energy, enthalpy, entropy, Gibbs energy, Helmholtz energy, specific heat, thermal conductivity, and viscosity of dry air. The results are compared with the perfect gas EoS and the Eq.s developed by The International Association for the Properties of Water and Steam (IAPWS).
\end{abstract}

\section{Keywords: Thermodynamic properties of dry air, thermophysical properties of dry air, Peng-Robison EoS, cubic EoS.}

\section{Introduction}

Usually, perfect gas Eq. of state (EoS) is used in order to calculate thermodynamic and thermophysical properties of dry air because of its simplicity. It might be suitable for many applications; however, error level will increase if highpressure applications are in the question. An Eq. of state with better accuracy of thermodynamic properties will be required for extreme cases. Moreover, in thermophysical property predictions, the approach is to assume such properties as only the function of temperature. But properties like viscosity and thermal conductivity heavily depend on pressure as well as temperature. Furthermore, such properties are quite a nonlinear function of pressure so that a linear interpolation type of correction of properties will not be correct.

Cubic Eq. of states are basically for the gas phase and can be accurate enough for high pressure applications as well. It is also possible to solve inverse Eq. $\mathrm{v}(\mathrm{T}, \mathrm{P})$ avoiding of more complex iterative root finding process.

In this paper, Peng-Robinson model is used for critical properties and acentric factor for pure gases. The mixing rule proposed by Harstad et al. is used to extend the PengRobinson EoS to mixtures [1] and dry air (nitrogen, oxygen, argon, carbon dioxide, neon, helium, methane, krypton, hydrogen and xenon09 [2]. Peng-Robinson EoS model was suggested by Ding-Yu Peng and Donald B. Robinson in 1976 to achieve simple and accurate predictions such as the compressibility factor liquid phase [3-4]. Since 1976, it has been widely used in thermodynamic calculations in industrial and scientific studies [5].

The objective of this study is propose an accurate EoS for dry air compared to the ideal gas EoS for thermodynamic and heat transfer analyse. Set of computer programs were developed by using Peng-Robinson EoS for determining specific volume, internal energy, enthalpy, entropy, Gibbs energy, Helmholtz energy, specific heat, thermal conductivity, and viscosity of dry air. The results were compared with the perfect gas EoS and others EoSs developed by The International Association for the Properties of Water and Steam (IAPWS) [6].

\section{Methodology \\ 2.1 Formulation of Eq. of State}

Peng-Robinson cubic Eq. of state was considered for dry exhaust gas mixture in this paper. Parameters of the Eq. is defined in terms of the critical properties and the acentric factor. Cubic Eq. of state has a general form of Eq. as [7] $P=\frac{R T}{v-b}-\frac{a}{v^{2}+u b v+w b^{2}}$

Peng-Robinson EoS coefficients: $\mathrm{u}=2, \mathrm{w}=-1$ so that Eq. took the form:

$P=\frac{R T}{v-b}-\frac{a}{v^{2}+2 b v-b^{2}}$

where

$b=\frac{0.0780 R T_{\text {crit }}}{P_{\text {crit }}}$

$a=\frac{0.45724 R^{2} T_{\text {crit }}^{2}}{P_{\text {crit }}}\left[1+f \omega\left(1-T_{r}^{0.5}\right)\right]^{2}$

$f \omega=0.37464+1.54226 \omega-0.269992 \omega^{2}$

$\omega$ is the Pitzer's acentric factor calculated as:

$\omega=-\log _{10} P_{\text {saturated vapor }}\left(\right.$ at $\left.T_{r}=0.7\right)-1$

The reduced vapor pressure $\left(P_{r}=P / P_{\text {crit }}\right)$ at $T_{r}=$ $T / T_{\text {crit }}=0.7$ is necessary to obtain the values of $\omega$. The Eq. can be written in the following form as well:
$Z^{3}-\left(1+B^{*}-u B^{*}\right) Z^{2}+\left(A^{*}+w B^{* 2}-u B^{*}-u B^{* 2}\right) Z-$
$A^{*} B^{*}-B^{* 2}-w B^{* 2}-w B^{* 3}=0$
where
$A^{*}=\frac{a P}{R^{2} T^{2}}$ 
$\begin{aligned} B^{*} & =\frac{b P}{R T} \\ Z & =\frac{P v}{R T}\end{aligned}$

So far Eq.s given above are for a single gas only. We will use the Peng-Robinson EoS for dry air as a mixture of oxygen, nitrogen, argon, carbon dioxide, etc.. A detail list of the component gases and their mole fractions in dry air is given in Table 1. More recently, Harstad, Miller, and Bellan [1] have presented computationally efficient forms of EoS for gas mixtures, particularly of Peng-Robinson EoS. They have also shown that it is possible to extend the Eq.s' validity beyond the range of data using departure functions. In this study, the mixing rule proposed by Harstad et al. is used to extend the PR Eq. of state to mixtures [1]. In particular, the parameters $\mathrm{a}$ and $\mathrm{b}$ can be obtained by

$a=\sum_{i} \sum_{j} y_{i} y_{j} a_{i j}$

$b=\sum_{i} y_{i} b_{i}$

where $\mathrm{y}$ is the mole fraction in the vapor phase and

$b_{i j}=\frac{0.0780 R T_{\text {crit } i j}}{P_{\text {crit } i j}}$

$a_{i j}=\frac{0.45724 R^{2} T_{\text {crit } i j}^{2}}{P_{\text {crit } i j}}\left[1+f \omega_{i j}\left(1-T_{r i j}^{0.5}\right)\right]^{2}$

$f \omega_{i j}=0.37464+1.54226 \omega_{i j}-0.269992 \omega_{i j}^{2}$

$T_{\text {rij }}=T / T_{\text {crit } i j}$

The diagonal elements of the "critical coefficients" matrices are equal to their corresponding pure substance counterparts, i.e., $T_{\text {crit } i i}=T_{\text {crit } i}, P_{\text {crit } i i}=P_{\text {crit } i}$, and $\omega_{i i}=$ $\omega_{i}$. The off-diagonal elements are evaluated through additional rules:

$P_{\text {crit } i j}=\frac{Z_{\text {crit } i j} R T_{\text {crit } i j}}{V_{\text {crit } i j}}$

$V_{\text {crit } i j}=\frac{1}{8}\left[\left(V_{\text {crit } i i}\right)^{1 / 3}+\left(V_{\text {crit } j j}\right)^{1 / 3}\right]$

$Z_{\text {crit } i j}=\frac{1}{2}\left[Z_{\text {crit } i i}+Z_{\text {crit } j j}\right]$

$\omega_{i j}=\frac{1}{2}\left[\omega_{i i}+\omega_{j j}\right]$

$T_{\text {crit } i j}=\sqrt{T_{\text {crit } i i} T_{\text {crit } j j}}\left(1-k_{i j}\right)$

where interaction coefficient $k_{i j}$ can be calculated as:

$k_{i j}=1-\frac{\left(V_{\text {crit } i i} V_{\text {crit } j j}\right)^{1 / 2}}{V_{\text {crit } i j}}$

Partial derivatives with respect to a are

$\frac{\partial a}{\partial T}=-\frac{1}{T} \sum_{i} \sum_{j}\left(y_{i} y_{j} a_{i j} \frac{f \omega_{i j} \sqrt{T_{r i j}}}{1+f \omega_{i j}\left(1-\sqrt{T_{r i j}}\right)}\right)$

$\frac{\partial^{2} a}{\partial T^{2}}=\frac{0.457236 R^{2}}{2 T} \sum_{i} \sum_{j}\left(y_{i} y_{j} a_{i j}\left(1-f \omega_{i j}\right) \frac{T_{\text {crit } i j}}{P_{\text {crit } i j}} \sqrt{T_{r i j}}\right)$

The basic formulas to calculate cubic roots analytically are as follows (Tartaglia \& Cardano (1530)):

$y=a_{0}+a_{1} x+a_{2} x^{2}+a_{3} x^{3}$

$a=a_{2} / a_{3} \quad b=a_{1} / a_{3} \quad c=a_{0} / a_{3}$

$y=c+b x+a x^{2}+x^{3}$

$Q=\frac{a^{2}-3 b}{9} \quad z=2 a^{3}-9 a b+27 c$

$R=z / 54$

$$
\begin{gathered}
\left\{\begin{array}{c}
\theta=\cos ^{-1}\left(\frac{R}{\sqrt{Q^{3}}}\right) \\
x_{0}=-2 \sqrt{Q} \cos [\theta / 3]-a / 3 \\
x_{1}=2 \sqrt{Q} \cos [(\theta-2 \pi) / 3]-a / 3 \\
x_{2}=2 \sqrt{Q} \cos [(\theta-2 \pi) / 3]-a / 3
\end{array}\right\} \\
\text { else }\left\{\begin{array}{c}
A=-\left(R+\sqrt{R^{2}-Q^{3}}\right)^{1 / 3} \\
i f(a==0) B=0 \\
e l s e B=Q / A \\
x_{1}=\left[\left(-\frac{A+B}{2}\right)-a / 3\right]+\left[\frac{\sqrt{3(A-B)}}{2}\right] \\
x_{0}=(A+B-a / 3) \\
x_{2}=\left[\left(-\frac{A+B}{2}\right)-a / 3\right]-\left[\frac{\sqrt{3(A-B)}}{2}\right]
\end{array}\right\}
\end{gathered}
$$

To solve the Peng-Robinson EoS for dry air as a mixture of gases, the specific heat, $C_{p}(T)$, for each component gas is required. This is obtained from NIST (National Institute of Standards and Technology) thermochemical tables [7]. Since $C_{p}(T)$ value is for the ideal gas, ideal gas mixing rule applied to establish $C_{p}(T)$ value of the mixture from the given gases. For each individual gas, the following partial difference curve fitting formula is used.

$C_{p i}(T)=A_{i}+B_{i} 10^{-3} T+\frac{C_{i} 10^{5}}{T^{2}}+D_{i} 10^{-6} T^{2} \quad T_{L i} \leq T \leq T_{H i}$

Component mole fractions and critical properties of dry air are given in Table 1 .

Table 1. Composition and critical properties of dry air.

\begin{tabular}{llllll}
\hline Name & Mol \% & $\mathrm{T}_{\mathrm{c}}$ & $\mathrm{P}_{\mathrm{c}}$ & $\mathrm{Z}_{\mathrm{c}}$ & $\omega$ \\
\hline Nitrogen & 78.084 & 126.2 & 33.9 & 0.29 & 0.039 \\
Oxygen & 20.946 & 154.6 & 50.4 & 0.288 & 0.025 \\
Argon & 0.934 & 150.8 & 48.7 & 0.291 & 0.001 \\
Carbon dioxide & 0.0397 & 304.1 & 73.8 & 0.274 & 0.239 \\
Neon & 0.001818 & 44.4 & 27.6 & 0.311 & -0.029 \\
Helium & 0.000524 & 5.19 & 2.27 & 0.302 & -0.365 \\
Methane & 0.000179 & 190.4 & 46 & 0.288 & 0.011 \\
Krypton & 0.000001 & 209.4 & 55 & 0.288 & 0.005 \\
Hydrogen & 0.0000005 & 33 & 12.9 & 0.303 & -0.216 \\
Xenon & 0.00000009 & 289.7 & 58.4 & 0.287 & 0.008 \\
\hline
\end{tabular}

The values of $\mathrm{C}_{\mathrm{p}}$ are predicted from Eq. (2.31) and the values for nitrogen and oxygen are shown in Figures 1 and 3 respectively. The error values are given in Figures 2 and 4 . The curve fitting coefficents of argon, nitrogen and oxygen are given in Table 2, 3 and 4, respectively.

Table 2. Cp (kJ/kmol K) partial continuous curve fitting Eq. constants for Argon.

\begin{tabular}{lllllll}
\hline $\mathrm{i}$ & $\mathrm{Ai}$ & $\mathrm{Bi}$ & $\mathrm{Ci}$ & $\mathrm{Di}$ & $\mathrm{T}_{\mathrm{L}, \mathrm{i}}$ & $\mathrm{T}_{\mathrm{H}, \mathrm{i}}$ \\
\hline 0 & 20.786 & $0.00 \mathrm{E}+00$ & $0.00 \mathrm{E}+00$ & $0.00 \mathrm{E}+00$ & 100 & 6000
\end{tabular}




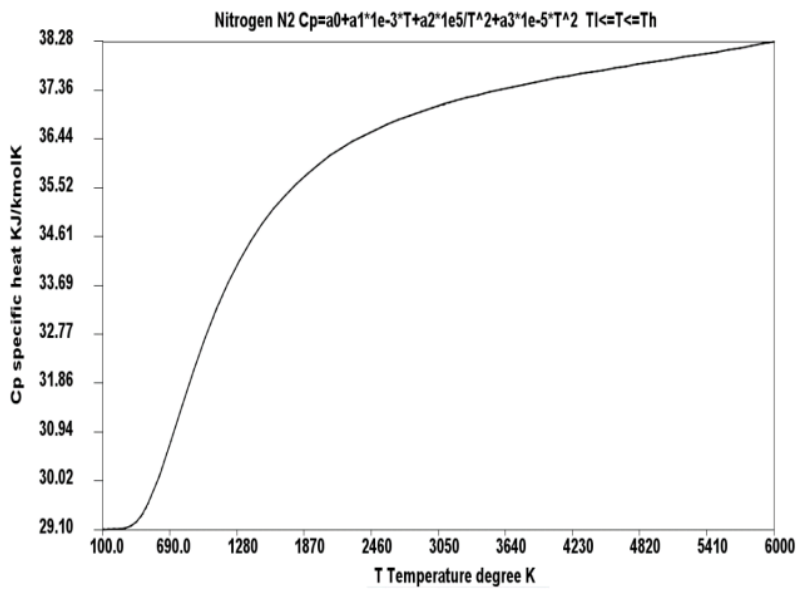

Figure 1. Change of $C_{P}$ with temperature for Nitrogen $\mathrm{kJ} /(\mathrm{kmol} \mathrm{K})$.

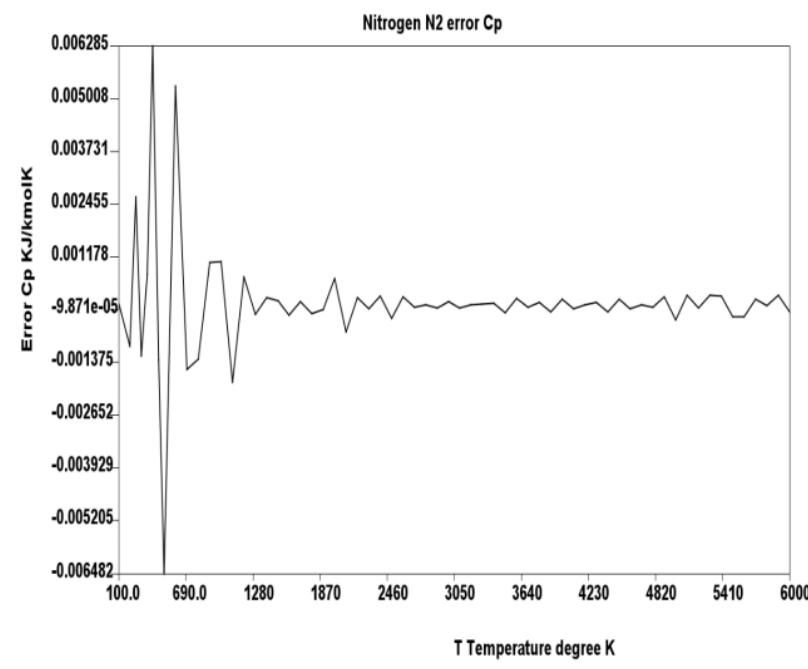

Figure 2. Change of error levels of predictions with temperature of $C_{p}$ in Nitrogen $\mathrm{kJ} /(\mathrm{kmol} \mathrm{K})$.

Table 3. Cp $(\mathrm{kJ} / \mathrm{kmol} \mathrm{K})$ curve fitting Eq. constants for Nitrogen.

\begin{tabular}{lllllll}
\hline $\mathrm{i}$ & $\mathrm{Ai}$ & $\mathrm{Bi}$ & $\mathrm{Ci}$ & $\mathrm{Di}$ & $\mathrm{T}_{\mathrm{L}, \mathrm{i}}$ & $\mathrm{T}_{\mathrm{H}, \mathrm{i}}$ \\
\hline 0 & 29.40863 & -2.25144 & -0.01247 & 4.52088 & 100 & 350 \\
1 & 27.64616 & 0.88235 & 0.77007 & 4.76442 & 350 & 700 \\
2 & 21.60170 & 14.87841 & 3.81280 & -4.16546 & 700 & 1200 \\
3 & 29.83076 & 5.42156 & -15.04309 & -1.08961 & 1200 & 1700 \\
4 & 35.47674 & 0.97358 & -42.54762 & -0.09746 & 1700 & 2200 \\
5 & 34.92820 & 1.31940 & -38.18419 & -0.15991 & 2200 & 2700 \\
6 & 36.26252 & 0.58150 & -50.89836 & -0.04573 & 2700 & 3200 \\
7 & 35.65734 & 0.76616 & -34.66593 & -0.05981 & 3200 & 3700 \\
8 & 36.41804 & 0.43259 & -44.18470 & -0.02015 & 3700 & 4200 \\
9 & 38.07768 & -0.15296 & -80.31180 & 0.03679 & 4200 & 4700 \\
10 & 37.76028 & -0.04994 & -73.10115 & 0.02776 & 4700 & 5200 \\
11 & 39.97385 & -0.85455 & -77.57593 & 0.10125 & 5200 & 6000 \\
\hline
\end{tabular}

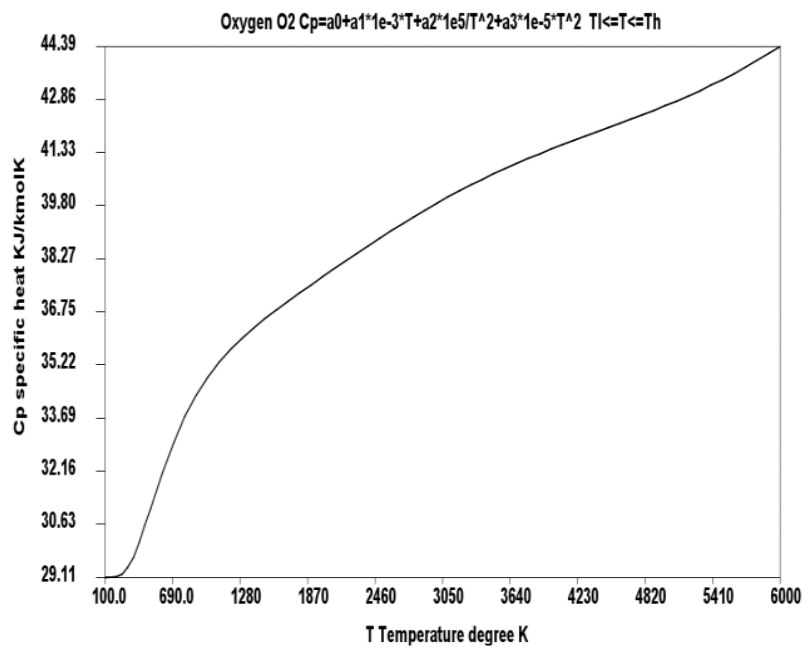

Figure 3. Change of $C_{P}$ with temperature for Oxygen $\mathrm{kJ} /(\mathrm{kmol} \mathrm{K})$.

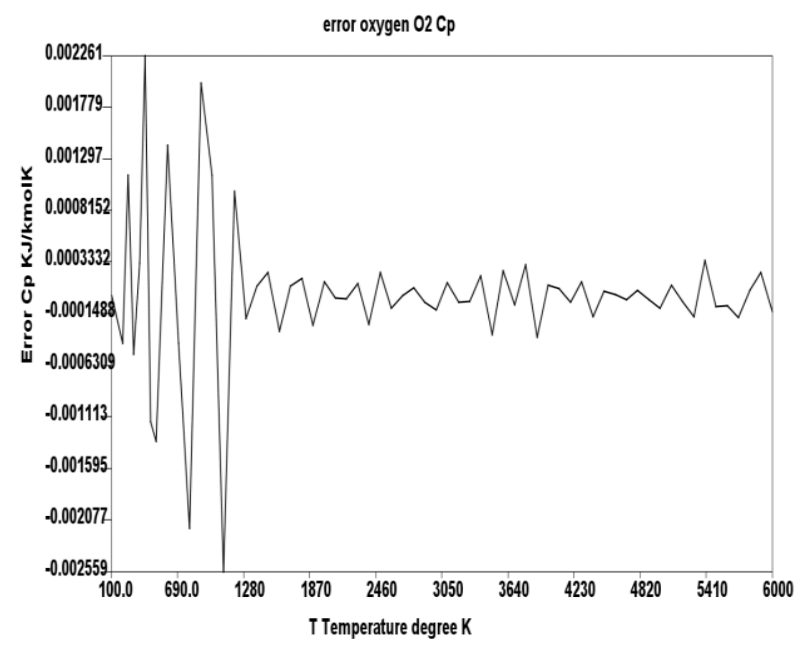

Figure 4. Change of error levels of predictions with temperature of $C_{p}$ in Oxygen $\mathrm{kJ} /(\mathrm{kmol} \mathrm{K})$.

Table 4. Cp (kJ/kmol K) curve fitting Eq. constants for Oxygen.

\begin{tabular}{lllllll}
\hline $\mathrm{i}$ & $\mathrm{Ai}$ & $\mathrm{Bi}$ & $\mathrm{Ci}$ & $\mathrm{Di}$ & $\mathrm{T}_{\mathrm{L}, \mathrm{i}}$ & $\mathrm{T}_{\mathrm{H}, \mathrm{i}}$ \\
\hline 0 & 30.43604 & -11.22375 & -0.04709 & 26.32148 & 100 & 350 \\
1 & 21.00131 & 23.61241 & 2.04654 & -10.13517 & 350 & 700 \\
2 & 29.74259 & 7.978910 & -6.12334 & -2.24031 & 700 & 1200 \\
3 & 36.31276 & 0.050814 & -19.65528 & 0.45704 & 1200 & 1700 \\
4 & 33.34540 & 2.32860 & -4.52664 & -0.03714 & 1700 & 2200 \\
5 & 31.03636 & 3.80481 & 11.95127 & -0.30141 & 2200 & 2700 \\
6 & 29.75055 & 4.50498 & 24.83303 & -0.40859 & 2700 & 3200 \\
7 & 32.14685 & 3.46653 & -12.25985 & -0.28271 & 3200 & 3700 \\
8 & 43.52700 & -0.66976 & -268.79475 & 0.14080 & 3700 & 4200 \\
9 & 54.76822 & -4.21849 & -604.90568 & 0.45650 & 4200 & 4700 \\
10 & 63.26081 & -6.72640 & -894.08145 & 0.66490 & 4700 & 5200 \\
11 & 71.31479 & -8.89365 & -1220.2917 & 0.82844 & 5200 & 6000 \\
\hline
\end{tabular}

\subsection{Formulations of Thermodynamic Properties}

Now we can establish other thermodynamic properties of dry air which is not given directly by the Peng-Robinson EoS. Entropy of dry air is expressed as

$$
\begin{aligned}
& d s=\frac{C_{v}(T)}{T} d T+\left(\frac{\partial P(T, v)}{\partial T}\right)_{v} d v \\
& d s=\frac{R-C_{p}(T)}{T} d T+\left(\frac{\partial P(T, v)}{\partial T}\right)_{v} d v
\end{aligned}
$$


$S(T, V)=s_{0}+\int_{T_{0}}^{T} \frac{C_{p}(T)-R}{T} d T+\int_{v_{0}}^{v}\left(\frac{\partial P}{\partial T}\right)_{v} d v$

The last term of Eq. (2.34) can be evaluated as

$\int_{v_{0}}^{v}\left(\frac{\partial P}{\partial T}\right)_{v} d v=\int_{v_{0}}^{v}\left[\frac{R}{v-b}-\left(\frac{1}{v^{2}+2 b v-b^{2}}\right)\left(\frac{\partial a}{\partial T}\right)_{v}\right] d v=$

$\int_{v_{0}}^{v}\left[\frac{R}{v-b}\right] d v-\left(\frac{\partial a}{\partial T}\right) \int_{v}^{v}\left(\frac{1}{v_{0}}\right) d v$

$\int_{v_{0}}^{v}\left(\frac{1}{v^{2}+2 b v-b^{2}}\right) d v=\int_{v_{0}}^{v}\left(\frac{1}{(v+b)^{2}-2 b^{2}}\right) d v$

$X=(v+b) \quad A=\sqrt{2} b$

$\int \frac{1}{X^{2}-A^{2}} d X=\int \frac{1}{(X-A)(X+A)} d X=\frac{1}{2 A}\left[\int \frac{1}{X-A} d X+\right.$

$\left.\int \frac{1}{X+A} d X\right]$

$\int_{v_{0}}^{v}\left(\frac{1}{v^{2}+2 b v-b^{2}}\right) d v=\frac{1}{2 \sqrt{2} b}\left[\ln \frac{(v+b-\sqrt{2} b)}{(v+b+\sqrt{2} b)}-\ln \frac{\left(v_{0}+b-\sqrt{2} b\right)}{\left(v_{0}+b+\sqrt{2} b\right)}\right]$

$\left(\frac{\partial P}{\partial T}\right)_{v}=\frac{R}{v-b}-\frac{\partial}{\partial a}\left(\frac{a}{v^{2}+2 b v-b^{2}}\right)\left(\frac{\partial a}{\partial T}\right)_{v}=\frac{R}{v-b}-$ $\left(\frac{1}{v^{2}+2 b v-b^{2}}\right)\left(\frac{\partial a}{\partial T}\right)_{v}$

Internal energy of dry air can be derived similarly as

$d u=C_{v}(T) d T+\left(T\left(\frac{\partial P(T, v)}{\partial T}\right)_{v}-P(T, v)\right) d v$

$d u=\left(R-C_{p}(T)\right) d T+\left(T\left(\frac{\partial P(T, v)}{\partial T}\right)_{v}-P(T, v)\right) d v$

$u(T, v)=u_{0}+\int_{T_{0}}^{T}\left(C_{p}(T)-R\right) d T+\int_{v_{0}}^{v}\left(T\left(\frac{\partial P(T, v)}{\partial T}\right)_{v}-\right.$

$P(T, v)) d v$

Second integral part of the Eq. 2.43 can be evaluated as

$\int_{v_{0}}^{v}\left[T\left(\frac{\partial P}{\partial T}\right)_{v}-P\right]=\int_{v_{0}}^{v}\left[\frac{R T}{v-b}-\left(\frac{1}{v^{2}+2 b v-b^{2}}\right) T\left(\frac{\partial a}{\partial T}\right)_{v}-\left(\frac{R T}{v-b}-\right.\right.$

$\left.\left.\frac{a}{v^{2}+2 b v-b^{2}}\right)\right] d v=\int_{v_{0}}^{v}\left[\left[a-T\left(\frac{\partial a}{\partial T}\right)_{v}\right]\left(\frac{1}{v^{2}+2 b v-b^{2}}\right) T\left(\frac{\partial a}{\partial T}\right)_{v}\right] d v$

$\int_{v_{0}}^{v}\left[T\left(\frac{\partial P}{\partial T}\right)_{v}-P\right]=\frac{\left[a-T\left(\frac{\partial a}{\partial T}\right)_{v}\right]}{2 \sqrt{2} b}\left[\ln \frac{(v+b-\sqrt{2} b)}{(v+b+\sqrt{2} b)}-\ln \frac{\left(v_{0}+b-\sqrt{2} b\right)}{\left(v_{0}+b+\sqrt{2} b\right)}\right]$

Enthalpy of dry air is expressed as

$h(T, v)=u+v P(T, v)$

$h(T, v)=h(T, v)-T P(T, v)$

Specific heat at constant pressure is expressed as

$C_{p}(T)=\sum_{i=0}^{n-1} y_{i} C_{p i}(T)=\sum_{i=0}^{n-1} y_{i}\left[A_{i}+B_{i} 10^{-3} T+\right.$

$\left.\frac{C_{i} 10^{5}}{T^{2}}+D_{i} 10^{-6} T^{2}\right] \quad T_{L 0} \leq T \leq T_{H 0}$

Specific heat at constant volume is expressed as

$C_{v}(T)=C_{p}(T)-R=\sum_{i=0}^{n-1} y_{i} C_{v i}(T)$

Where $C_{v i}(T)$ is the specific heat at constant volume of each component gases.

$$
\begin{aligned}
& C_{v}(T)=\sum_{i=0}^{n-1} y_{i}\left(A_{i}-R+B_{i} 10^{-3} T+\frac{C_{i} 10^{5}}{T^{2}}+\right. \\
& \left.D_{i} 10^{-6} T^{2}\right)
\end{aligned}
$$

$\int_{T L_{0}}^{T} C_{v}(T) d T=\sum_{i=0}^{m-1}\left[\left(A_{i}-R\right)\left(T_{H i}-T_{L i}\right)+\frac{B_{i}}{2} 10^{-3}\left(T_{H i}^{2}-\right.\right.$

$\left.\left.T_{L i}^{2}\right)-C_{i} 10^{5}\left(\frac{1}{T_{H i}}-\frac{1}{T_{L i}}\right)+\frac{D_{i} 10^{-6}}{3}\left(T_{H i}^{3}-T_{L i}^{3}\right)\right]+\left[A_{m}\left(T-T_{L m}\right)+\right.$

$\left.\frac{B_{m}}{2} 10^{-3}\left(T^{2}-T_{L m}^{2}\right)-C_{m} 10^{5}\left(\frac{1}{T}-\frac{1}{T_{L m}}\right)+\frac{D_{m} 10^{-6}}{3}\left(T^{3}-T_{L m}^{3}\right)\right]$

for $T_{0}=T_{L 0}=100 \mathrm{~K}$ and $T_{L m} \leq T \leq T_{H m}$

$\int_{T_{0}}^{T} C_{v}(T) d T=\sum_{i=0}^{n-1} y_{i} \int_{T_{0}}^{T} C_{v i}(T) d T$

for $T_{0}=T_{L 0}=100 \mathrm{~K}$ and $T_{L m} \leq T \leq T_{H m}$

$\int_{T_{0}}^{T} \frac{C_{v}(T) d T}{T}=\sum_{i=0}^{n-1} y_{i} \int_{T_{0}}^{T} \frac{C_{v i}(T) d T}{T}$

To adopt Eqs. (2.52) and (2.53) to any other reference point $\mathrm{T}_{\mathrm{r}}$

$\int_{T_{r}}^{T} C_{v i}(T) d T=\int_{T_{0}}^{T} C_{v i}(T) d T-\int_{T_{0}}^{T_{r}} C_{v i}(T) d T$

$\int_{T_{r}}^{T} \frac{C_{v i}(T)}{T} d T=\int_{T_{0}}^{T} \frac{C_{v i}(T)}{T} d T-\int_{T_{0}}^{T_{r}} \frac{C_{v i}(T)}{T} d T$

\subsection{Formulations of Thermophysical Properties}

To estimate the thermal conductivity and viscosity of dry air, equations suggested by Kadoya et al [8] are used.

$\eta\left(T_{r}, \rho_{r}\right)=H\left[\eta_{0}\left(T_{r}\right)+\Delta \eta\left(\rho_{r}\right)\right]$

where

$\eta_{0}\left(T_{r}\right)=A_{0} T_{r}+A_{1} T_{r}^{0.5}+A_{2}+\frac{A_{3}}{T_{r}}+\frac{A_{4}}{T_{r}^{2}}+\frac{A_{5}}{T_{r}^{3}}+\frac{A_{6}}{T_{r}^{4}}$

As it can be seen in Eq. (2.56), the $\eta_{0}$ (low pressure viscosity) depends on only temperature, and the $\Delta \eta$ is the difference between low and high pressure viscosity

$\Delta \eta\left(\rho_{r}\right)=\sum_{i=1}^{4} B_{i} \rho_{r}^{i}$

There is a similar approach for thermal conductivity.

$\mathrm{k}\left(T_{r}, \rho_{r}\right)=\Lambda\left[k_{0}\left(T_{r}\right)+\Delta \mathrm{k}\left(\rho_{r}\right)\right]$

where

$k_{0}\left(T_{r}\right)=C_{0} T_{r}+C_{1} T_{r}^{0.5}+C_{2}+\frac{C_{3}}{T_{r}}+\frac{C_{4}}{T_{r}^{2}}+\frac{C_{5}}{T_{r}^{3}}+\frac{C}{T_{r}^{4}}$

$\Delta \mathrm{k}\left(\rho_{r}\right)=\sum_{i=1}^{4} D_{i} \rho_{r}^{i}$

$\rho_{r}=\rho / \rho^{*} \quad T_{r}=T / T^{*}$

In Eqs. (2.55) to (2.60), $\rho^{*}$ is equal to $314.3 \mathrm{~kg} / \mathrm{m}^{3}$, $\Lambda$ is equal to $25.9778\left(10^{-3} \mathrm{~W} /(\mathrm{mK}), \mathrm{H}\right.$ is equal to 6.1609 (10-6 Pas) and $T^{*}$ is equal to $132.5 \mathrm{~K}$. The coefficients of Eqs. (2.55) to (2.60) are given in Table 5.

Table 5. Coefficients of Eqs.(2.55) to (2.60). [8]

\begin{tabular}{lllll}
\hline $\mathrm{i}$ & $\mathrm{A}_{\mathrm{i}}$ & $\mathrm{B}_{\mathrm{i}}$ & $\mathrm{C}_{\mathrm{i}}$ & $\mathrm{D}_{\mathrm{i}}$ \\
\hline 0 & 0.128517 & 0.465601 & 0.239503 & 0.402287 \\
1 & 2.60661 & 1.26469 & 0.006497 & 0.356603 \\
2 & -1 & -0.511425 & 1 & -0.163159 \\
3 & -0.709661 & 0.2746 & -1.92615 & 0.138059 \\
4 & 0.662534 & & 2.00383 & -0.020172 \\
5 & -0.197846 & & -1.07553 & \\
6 & 0.0077014 & & 0.229414 &
\end{tabular}


Table 6. Additional properties derived from PengRobinson EoS

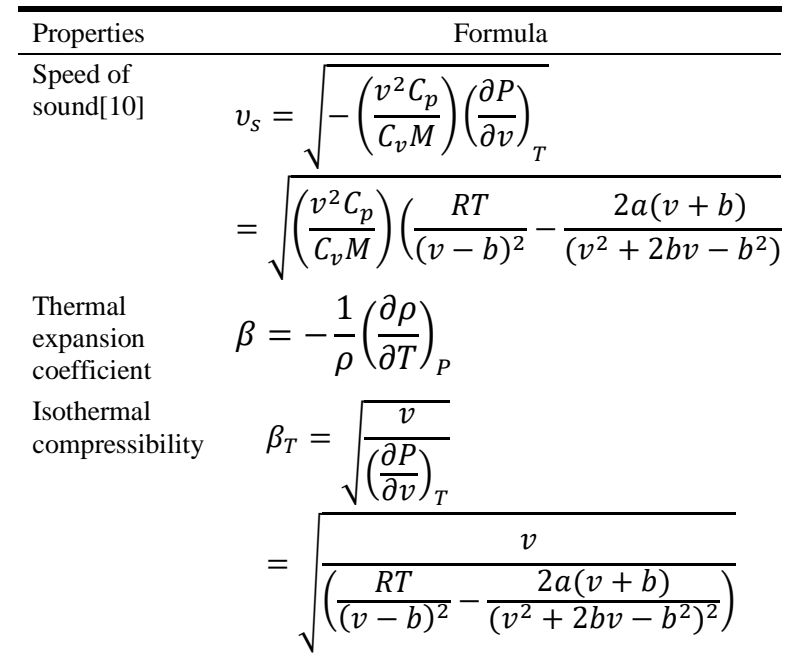

\section{Results}

Several programs were developed in java language to carry out the predictions. The names of programs are given in Table 6. Air_IAPWS was developed by using IAPWS formulations [6] and air_PG is for the perfect gas EoS for air. The details of these programs are not described here, but they are given for comparison purposes.

Table 6. Programs developed for calculating dry air properties.

\begin{tabular}{ll}
\hline Model Name & Reference and Description \\
\hline air_IAPWS & IAPWS Formulation [6] \\
air_PR & Peng-Robinson EoS for mixtures [11] \\
air_PG & Perfect gas EoS as a single gas.
\end{tabular}

\begin{tabular}{|c|c|c|c|c|}
\hline PR & & & $\square$ & $x$ \\
\hline 0 & 1 & 2 & 3 & \\
\hline 0 & $\mathrm{P}$, pressure & 100.0 & $\mathrm{kPA}$ & \\
\hline 1 & $\mathrm{~T}$, temperature & 300.0 & $\operatorname{deg} \mathrm{K}$ & \\
\hline 2 & $\mathrm{v}$, specific volume & 0.86068925616902 & $\mathrm{~m}^{3} / \mathrm{kg}$ & \\
\hline 3 & $h$, enthalpy & 27.4978677275264 & $\mathrm{KJ} / \mathrm{kg}$ & \\
\hline 4 & $\mathrm{u}$, internal energy & 19.7667000505451. & $\mathrm{KJ} / \mathrm{kg}$ & \\
\hline 5 & s, entropy & 0.09428990072652 & $\mathrm{KJ} / \mathrm{kgK}$ & \\
\hline 6 & $\mathrm{x}$, quality & 2.0 & $\mathrm{~kg}$ vap $/ \mathrm{kg}$ mix & \\
\hline 7 & $\mathrm{Cp}$, specific heat at.. & 1.00484279166310 & $\mathrm{KJ} / \mathrm{kgK}$ & \\
\hline 8 & $\mathrm{Cv}$, specific heat at.. & 0.71779290736475 & $\mathrm{KJ} / \mathrm{kgK}$ & \\
\hline 9 & $\beta$ isobaric thermal ... & 0.00334399246971. & $1 / \mathrm{K}$ & \\
\hline 10 & $\mathrm{~K}$ isothermal comp... & 9.97509391205509 & $\mathrm{kPa}$ & \\
\hline 11 & $\eta$ Dynamic viscosity & 1.85581523427694 & $\mathrm{~Pa} . \mathrm{s}$ & \\
\hline 12 & $\mathrm{k}$ Thermal conduct... & 0.02623176260023 & $\mathrm{~W} / \mathrm{mK}$ & \\
\hline 13 & $\sigma$ Surface tension & 0.0 & $\mathrm{~N} / \mathrm{m}$ & \\
\hline 14 & Prandtl number & 0.71234042678339 & & \\
\hline 15 & $\rho$ density & $1.16185951298039 .$. & $\mathrm{kg} / \mathrm{m}^{\wedge} 3$ & \\
\hline 16 & speed of sound & 347.5475043590632 & $\mathrm{~m} / \mathrm{s}$ & \\
\hline
\end{tabular}

Figure 5. Peng-Robinson EoS program graphic output for $P=100 \mathrm{kPa}$ and $\mathrm{T}=300 \mathrm{~K}$.

Results obtained for several thermodynamic properties by using different EoSs are compared in Table 7.

Table 7. Comparisons of the three different EoS for dry air.

\begin{tabular}{lllllll}
\hline Model & $\begin{array}{c}\mathrm{P} \\
(\mathrm{kPa})\end{array}$ & $\begin{array}{l}\mathrm{T} \\
(\mathrm{deg} . \\
\mathrm{K})\end{array}$ & $\begin{array}{l}\mathrm{v} \\
\left(\mathrm{m}^{3} / \mathrm{kg}\right)\end{array}$ & $\begin{array}{l}\mathrm{h} \\
(\mathrm{kJ} / \mathrm{kg})\end{array}$ & $\begin{array}{l}\mathrm{u} \\
(\mathrm{kJ} / \mathrm{kg})\end{array}$ & $\begin{array}{l}\mathrm{s} \\
(\mathrm{kJ} /(\mathrm{kg} . \mathrm{K}))\end{array}$ \\
\hline air_PR & 100 & 300 & 0.86068 & 27.49786 & 19.76670 & 0.09428 \\
air_PG & 100 & 300 & 0.86114 & 27.01007 & 19.30280 & 0.09809 \\
air_IAPWS & 100 & 300 & 0.86088 & 27.01361 & 19.28572 & 0.09810
\end{tabular}

\begin{tabular}{lllllll} 
air_PR & 500 & 300 & 0.17178 & 26.32825 & 18.77558 & -0.36967 \\
air_PG & 500 & 300 & 0.17222 & 27.01007 & 19.30280 & -0.36388 \\
air_IAPWS & 500 & 300 & 0.17197 & 26.11119 & 18.48476 & -0.36653 \\
air_PR & 1000 & 300 & 0.08568 & 24.88375 & 17.54150 & -0.57105 \\
air_PG & 1000 & 300 & 0.08611 & 27.01007 & 19.30280 & -0.56285 \\
air_IAPWS & 1000 & 300 & 0.08587 & 24.99335 & 17.48330 & -0.56881 \\
air_PR & 3000 & 300 & 0.02832 & 19.30848 & 12.66731 & -0.89554 \\
air_PG & 3000 & 300 & 0.02870 & 27.01007 & 19.30280 & -0.87821 \\
air_IAPWS & 3000 & 300 & 0.02850 & 20.62849 & 13.47535 & -0.89723 \\
air_PR & 100 & 500 & 1.43560 & 230.79834 & 165.57513 & 0.61237 \\
air_PG & 100 & 500 & 1.43524 & 230.10497 & 164.98788 & 0.61632 \\
air_IAPWS & 100 & 500 & 1.43571 & 230.10855 & 164.89739 & 0.61633 \\
air_PR & 500 & 500 & 0.28741 & 230.17163 & 164.80265 & 0.15019 \\
air_PG & 500 & 500 & 0.28704 & 230.10497 & 164.98788 & 0.15434 \\
air_IAPWS & 500 & 500 & 0.28752 & 229.90383 & 164.50290 & 0.15356 \\
air_PR & 1000 & 500 & 0.14389 & 229.39896 & 163.84406 & -0.04900 \\
air_PG & 1000 & 500 & 0.14352 & 230.10497 & 164.98788 & -0.04462 \\
air_IAPWS & 1000 & 500 & 0.14400 & 229.65482 & 164.01187 & -0.04637 \\
air_PR & 3000 & 500 & 0.04822 & 226.42496 & 160.086668 & -0.36516 \\
air_PG & 3000 & 500 & 0.04784 & 230.10497 & 164.98788 & -0.35998 \\
air_IAPWS & 3000 & 500 & 0.04834 & 228.73108 & 162.07077 & -0.36553 \\
\hline
\end{tabular}

In the following figures, the Peng-Robinson EoS is compared with the perfect gas EoS and IAPWS for the dry air EoS. IAPWS equation is based on the experimental data. The results of Peng-Robinson EoS are falling between the results of perfect gas EoS and IAPWS. This is the expected behavior for such a general EoS based on acentric factor.

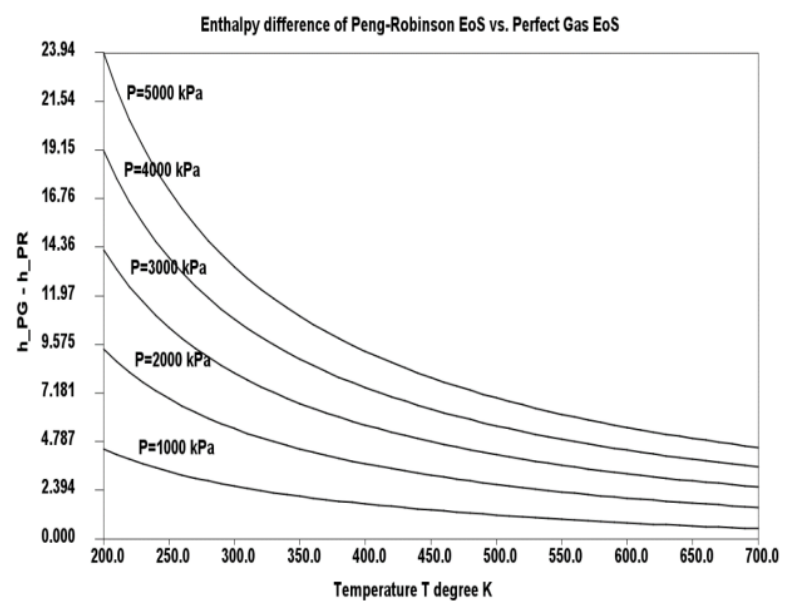

Figure 6. Enthalpy difference of Peng-Robinson and perfect gas EoS (for above $1000 \mathrm{kPa}$ ).

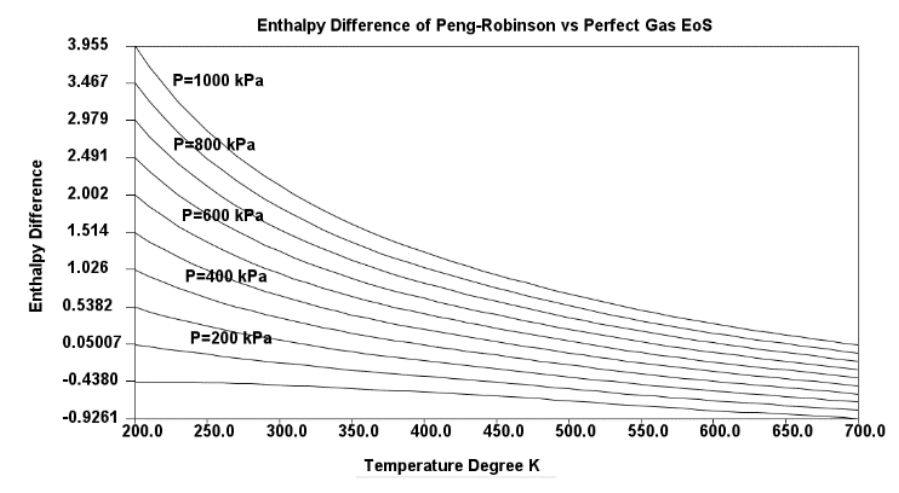

Figure 7. Enthalpy differences of Peng-Robinson and perfect gas EoS. 


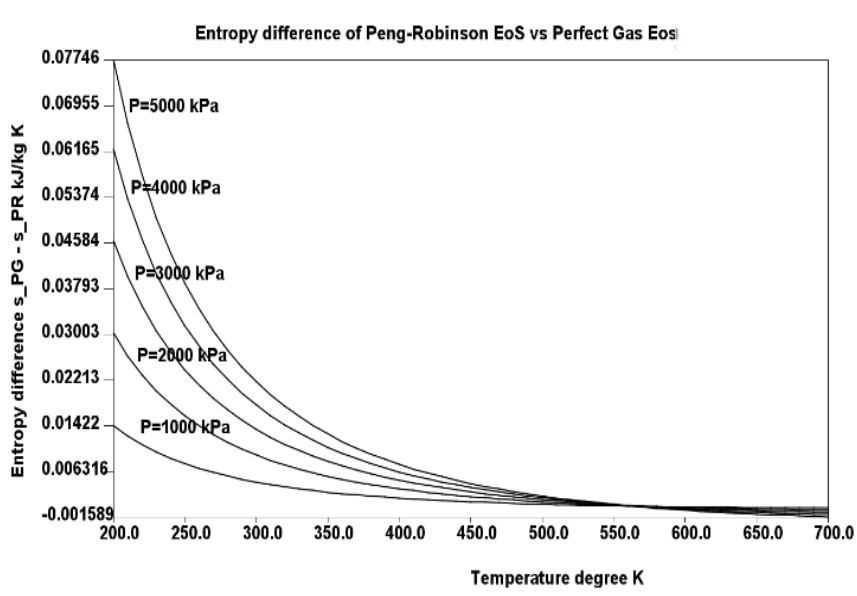

Figure 8. Entropy difference of Peng-Robinson and perfect gas EoS.

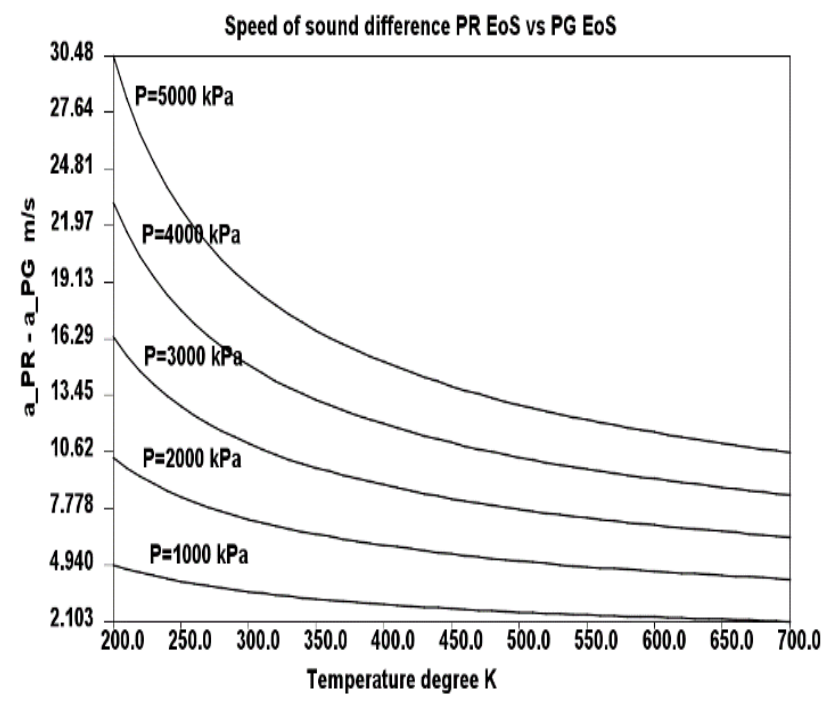

Figure 9. Speed of sound difference of Peng-Robinson and perfect gas EoS.

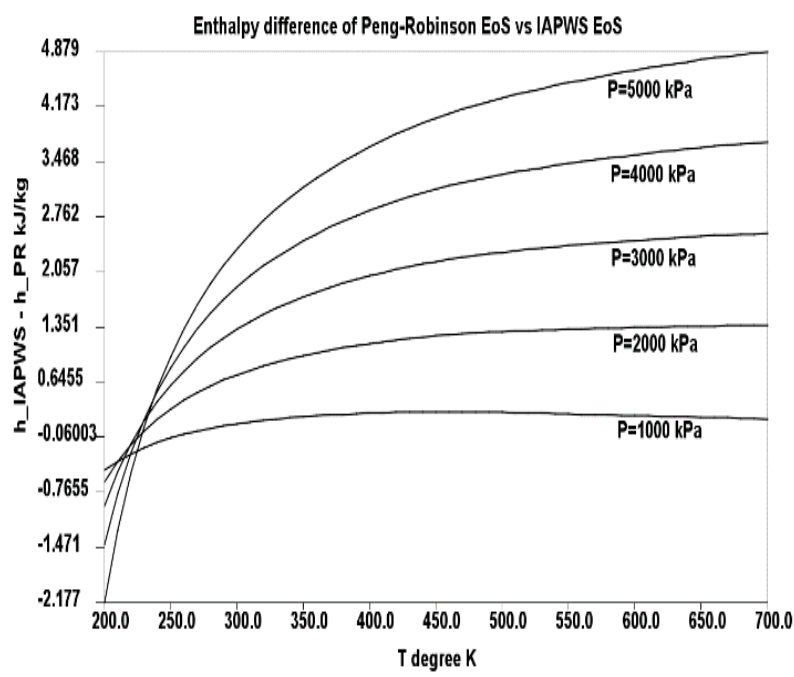

Figure 10. Enthalpy difference of Peng-Robinson and IAPWS EOS.

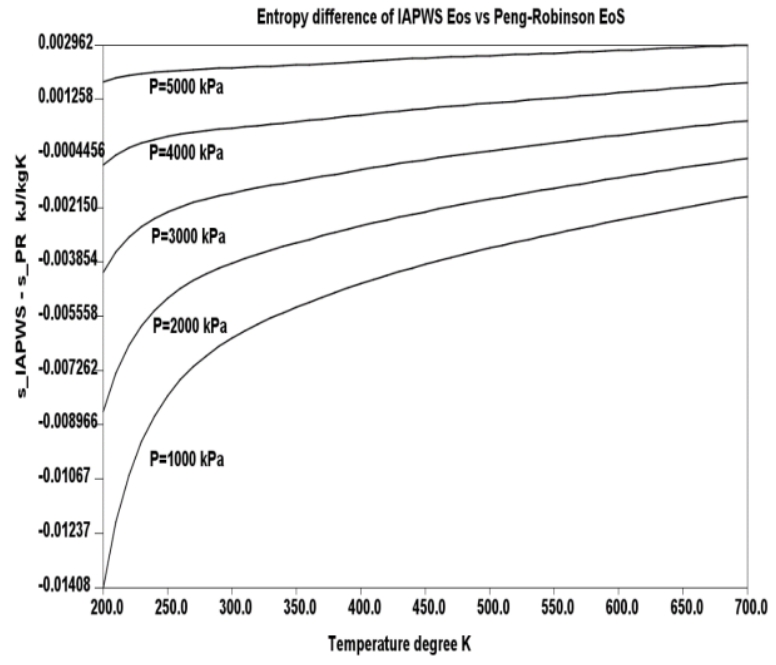

Figure 11. Entropy difference of Peng-Robinson and IAPWS EoS.

\section{Conclusions}

There are accurate EoS already available for air as a real gas. However, this package can be utilized as a calibration tool for a gas mixture to be used in combustion processes. Another basic application of such an EoS is to use it as a base to define humid air, which will be carried out by the authors in a separate publication.

Computer models for different set of real gas EoS by IAPWS and perfect gas formulations are also derived and results are compared. The results from the Peng-Robinson equation are between the results of perfect gas EoS and IAPWS. This is the expected for such a general EoS based on the acentric factor.

All the coefficients of EoSs for property estimations of dry air are given with details, which can be used for computational purposes. Whole set of computer codes developed in java programming language for researchers are available at: www.turhancoban.com.

\section{Nomenclature}

a Cubic Eq. coefficient $\left(\mathrm{Pa} \mathrm{kmol} / \mathrm{m}^{3}\right)$

b Cubic Eq. coefficient $\left(\mathrm{m}^{3} / \mathrm{kmol}\right)$

$\mathrm{C}_{\mathrm{p}} \quad$ Specific heat capacity at constant pressure (J/kg K)

$\mathrm{C}_{\mathrm{pi}} \quad$ Individual gas specific heat capacity at constant pressure $(\mathrm{J} / \mathrm{kg} \mathrm{K})$

$\mathrm{C}_{\mathrm{v}} \quad$ Specific heat capacity at constant volume (J/kg K)

$\mathrm{C}_{\mathrm{vi}} \quad$ Individual gas specific heat capacity at constant volume $(\mathrm{J} / \mathrm{kg} \mathrm{K})$

$\mathrm{F}_{\mathrm{w}} \quad$ A function of $\mathrm{w}$ specific for Peng-Robinson EoS

h Enthalpy $(\mathrm{kJ} / \mathrm{kg})$

$\mathrm{k}$ Thermal conductivity (W/m K)

M Molecular weight $(\mathrm{kg} / \mathrm{kmol})$

P Pressure $(\mathrm{kPa})$

R Universal gas constant $(8.314 \mathrm{~J} / \mathrm{mol} \mathrm{K})$

s Entropy $(\mathrm{kJ} / \mathrm{kg} \mathrm{K})$

$\mathrm{T}$ Temperature $(\mathrm{K})$

T* $132,5 \mathrm{~K}$

$\mathrm{u} \quad$ Internal energy $(\mathrm{kJ} / \mathrm{kg})$

$\mathrm{v} \quad$ Specific volume $\left(\mathrm{m}^{3} / \mathrm{kg}\right)$

$\mathrm{V} \quad$ Volume $\left(\mathrm{m}^{3}\right)$

Z Compressibility factor

Greek symbols

$\beta \quad$ Thermal expansion coefficient ( $1 / \mathrm{k})$

$\beta_{\mathrm{T}} \quad$ Isothermal compressibility

$\eta \quad$ Viscosity (Pa s) 


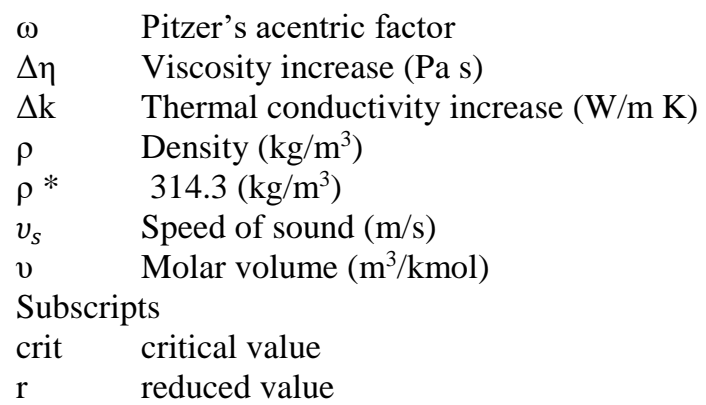

\section{References}

[1] K.G. Harstad, R.S. Miller and J. Bellan, "efficient highpressure state equation of states," AICHE Journal, Vol. 43, No. 6, pp. 1605-1610, 1997.

[2] E. W. Lemmon, R. T. Javobsen, "Viscosity and thermal conductivity eq.s for nitrogen, oxygen, argon, and air," International Journal of Thermophysics, Vol. 25, No. 1, January 2004.

[3] D. Peng and D. B. Robinson, "A new two-constant equation of state," Ind. Eng. Chem., Fundam., Vol. 15, No. 1, 1976.

[4] Z. Nasri, B. Housam "Applications of The PengRobinson equation of state using MATLAB," Chemical Engineering Education, 43, 2009.

[5] J.S. Lopez-Echeverry, S. Reif-Acherman, E. AraujoLopez "Peng-Robinson equation of state: 40 years through cubics," Fluid Phase Equilibria, 447, 39-71, 2017.
[6] The International Association for the Properties of Water and Steam, Guideline on an Eq. of State for Humid Air in Contact with Seawater and Ice, Consistent with the IAPWS Formulation 2008 for the Thermodynamic Properties of Seawater, Niagara Falls, Canada, IAPWS G8-10, 2010.

[7] R. C. Reid, J. M. Prausnitz, B. E. Poling, The Properties of Gases \& Liquids, Fourth Edition, McGraw-Hill, 1987.

[8] K. Kadoya, N. Matsunaga, and A. Nagashima, "Viscosity and thermal conductivity of dry air in the gaseous phase," Journal Physical Chemical Reference Data, 14, 947, 1985.

[9] NIST-JANAF Thermochemical Tables [Online]. Available: https://janaf.nist.gov/, (accessed May. 1, 2020).

[10] M. Salimi, A. Bahramian, "The Prediction of the Speed of Sound in Hydrocarbon Liquids and Gases: The PengRobinson Eq. of State Versus SAFT-BACK", Petroleum Science Technology, doi: 10.1080/10916466.2011.58030132:409-417, 2014.

[11] I. H. Bell, J. Wronski, S. Quailin, and V. Lemort, "Pure and pseudo-pure fluid thermophysical property evaluation and the open-source thermophysical property library CoolProp," Industrial \& Engineering Chemistry Research, doi: 10.1021/ie4033999, 2014. 